\title{
EQUILIBRIA AND STABILITY ANALYSIS OF A BRANCHED METABOLIC NETWORK WITH FEEDBACK INHIBITION
}

\author{
YACINe Chitour \\ Laboratoire des systèmes et signaux \\ Université Paris-Sud, CNRS, Supélec \\ 91192, Gif-sur-Yvette, France \\ FrÉdÉRIC GrognARD \\ Projet COMORE - INRIA \\ BP 9306902 Sophia-Antipolis Cedex, France \\ Georges Bastin \\ Centre for Systems Engineering and Applied Mechanics(CESAME) \\ Université Catholique de Louvain \\ Bâtiment Euler,4-6, avenue G.Lemaitre, \\ 1348 Louvain la Neuve, Belgium
}

\begin{abstract}
This paper deals with the analysis of a metabolic network with feedback inhibition. The considered system is an acyclic network of monomolecular enzymatic reactions in which metabolites can act as feedback regulators on enzymes located "at the beginning" of their own pathway, and in which one metabolite is the root of the whole network. We show, under mild assumptions, the uniqueness of the equilibrium. We then show that this equilibrium is globally attractive if we impose conditions on the kinetic parameters of the metabolic reactions. Finally, when these conditions are not satisfied, we show, with a specific fourth-order example, that the equilibrium may become unstable with an attracting limit cycle.
\end{abstract}

1. Introduction. The cellular metabolism is defined as the set of biochemical reactions that occur inside a living cell for growth and reproduction. It is usually represented by a large and intricate network connecting the involved biochemical species (called "metabolites"). The pathways of the network are called "metabolic pathways". In the metabolic engineering literature, it is widely accepted that "despite their immense complexity, metabolic systems are characterized by their ability to reach stable steady states" (quoted from [17, Chapter 4).

It should however be fair to recognize that a mathematical analysis of this fundamental stability property is a difficult question which was not fully investigated. A special difficulty comes from the existence of negative feedback inhibitions that are known for a long time to potentially induce instabilities and limit cycles. However, the analysis of the effect of feedback inhibition on the stability has been limited so far to unbranched pathways. In most studies, a single reaction in the pathway is supposed to be inhibited by the last metabolite only, whereas the other reactions

2000 Mathematics Subject Classification. 93B05, 93B60, 35P20.

Key words and phrases. Metabolic networks, equilibria, stability, small-gain. 
are considered to be not inhibited (e.g., [18, [10, [9, [13, [3]). In these contributions, the analysis generally relies on the existence of stable equilibria or limit cycles. More recently Alves and Savageau ([1]) have analyzed the stability of unbranched pathways with arbitrary patterns of multiple internal feedback inhibitions using the Routh stability criterion. They have in particular analyzed the effect of such internal inhibitions on the stability margin of the pathway.

In this paper, we shall extend the stability analysis to a more general class of branched metabolic networks made up of mono-molecular reactions. We shall assume that several end metabolites that appear in different branches inhibit a common step located before the first branch point. In order to perform the stability analysis, we shall use a specific mathematical technique which, to our knowledge, has not been used in previous papers on the stability of metabolic systems. This technique provides a sufficient condition under which the considered branched network has a single globally asymptotically stable equilibrium. We will mention also that this stability condition can alternatively be obtained with the approach of Angeli and Sontag ([2]) on interconnection of monotone systems.

The various modes of feedback inhibition in metabolic networks are briefly reviewed in [17, Chapter 5. In sequential feedback inhibition, the various branches of the network are decoupled (see Fig. 1.a); there is no inhibition by a metabolite belonging to one branch of the network acting on a reaction belonging to another branch, so that the different branches can be studied separately. The stability analysis of sequential feedback inhibition has been treated in our previous paper [4]. In the present paper, we consider the more complex case where several end-products inhibit the first reaction of a common step before the branch point. The inhibition can be operated by isofunctional enzymes which are selectively sensitive to one of the end-products (Fig 1.b); or a so-called cumulative inhibition can take place where a single enzyme uses multiple allosteric sites responding to each of the end-products (Fig 1.c). In this paper, we will give conditions for the stability of the equilibrium of such a network and show that inhibition, which is intuitively seen as regulation, can also lead to the destabilization of the system.

An example of such a network with inhibition, is the one given by the aspartate amino-acid pathways ([19, cited from [17]), see Figure 2. In this network, each produced amino-acid inhibits an enzyme of its own pathway. This action can be seen as a negative feedback, that regulates the behavior of the network. Indeed, if we, for example, consider a large excess of isoleucine $\left(X_{20}\right)$, the reaction $X_{16} \longrightarrow X_{17}$ is shut down, so that the concentration of isoleucine is progressively reduced.

The structure of the paper is as follows: in Section 2, a mass balance dynamical model of metabolic networks such as in Figure 1 will be presented. The equilibria of these models will then be studied in Section 3, followed by a stability analysis in Section 4, where global attractivity of a unique equilibrium is shown under some assumptions on the kinetic parameters. The non-genericity of the stability of the equilibrium is then illustrated in Section 5 with a specific simple fourth order example.

2. Model of a metabolic network. We consider a general class of branched metabolic networks made up of mono-molecular reactions of the form

$$
X_{s} \rightarrow X_{p}
$$




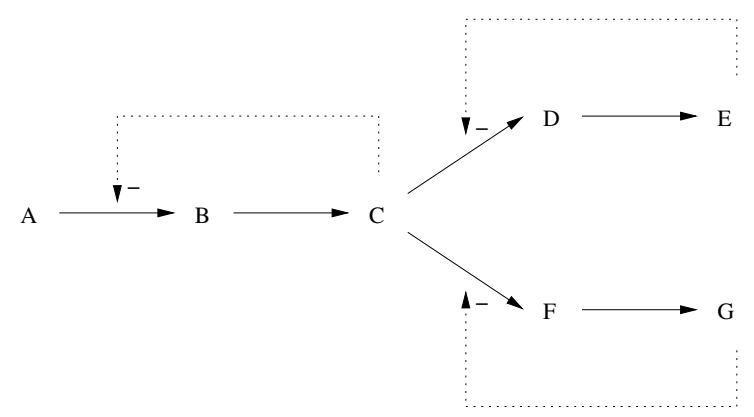

a) Sequential feedback inhibition

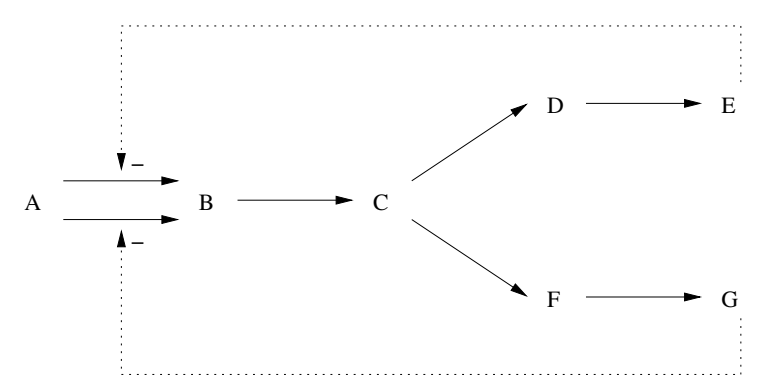

b) Feedback inhibition with isofunctional enzymes

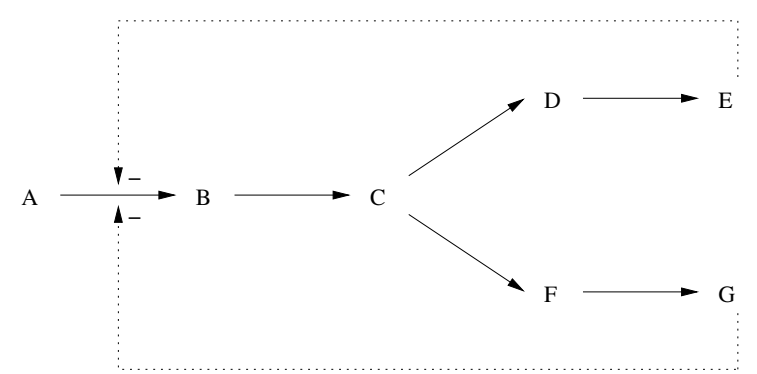

c) Cumulative feedback inhibition

Figure 1. Modes of feedback inhibition in metabolic networks (adapted from Stephanoupoulos et al. (1997)). The solid lines represent the reactions and the dotted lines the inhibitions.

The reaction can be inhibited by other metabolites of the network. The reaction rate of the reaction is denoted

$$
\varphi_{s p}\left(x_{s}, x^{[p]}\right),
$$

where $x_{s}$ denotes the concentration of the metabolite $X_{s}$ in the cell and $x^{[p]}$ denotes the vector containing the concentrations of the other metabolites that inhibit the reaction $X_{s} \rightarrow X_{p}$. Notice that the function $\varphi_{s p}$ may represent a sum of several parallel reactions $X_{s} \rightarrow X_{p}$ which are catalyzed by different isofunctional enzymes. 


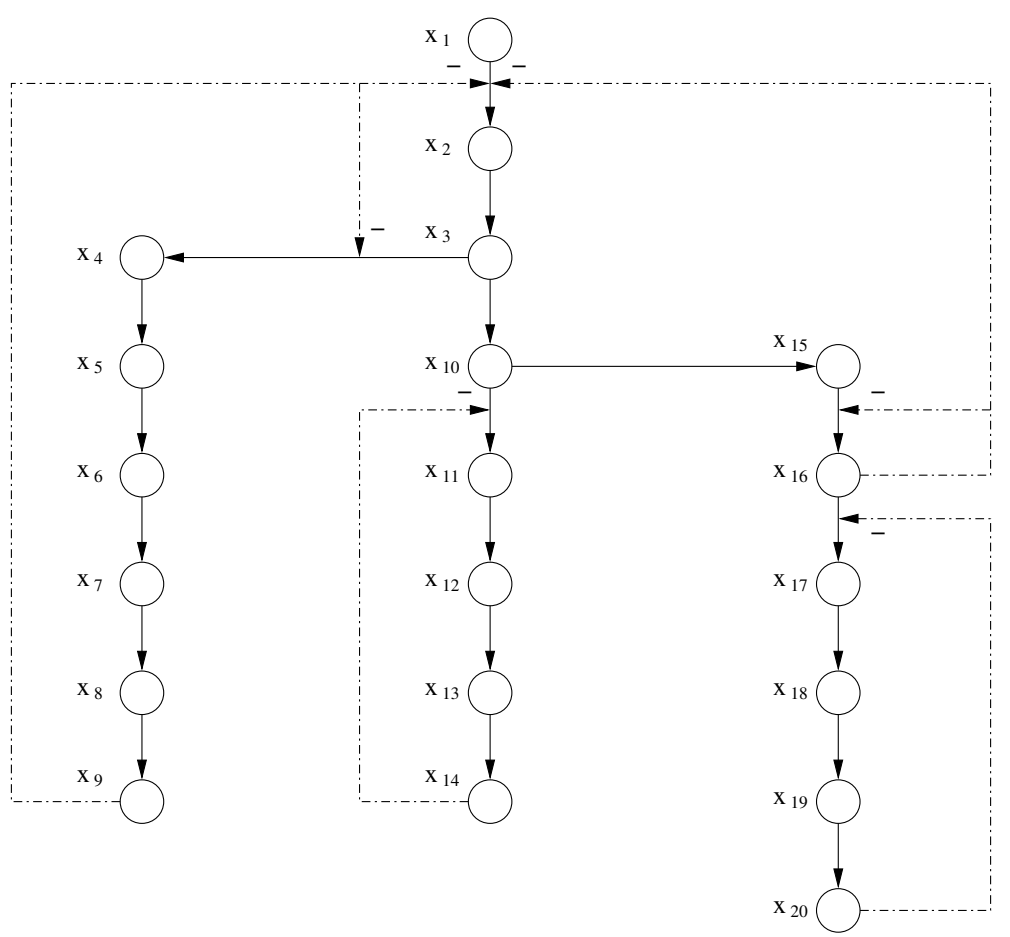

FIGURE 2. Metabolic network representing the aspartate aminoacid pathways: the solid lines represent the reactions and the dashdotted lines the inhibition produced by the state at the start of the arrow onto the reaction that lies at the end of the arrow. The root of the metabolic pathway is $x_{1}$ (aspartate), and the products are the corresponding amino acids: lysine $\left(x_{9}\right)$, methionine $\left(x_{14}\right)$, threonine $\left(x_{16}\right)$, and isoleucine $\left(x_{20}\right)$.

In addition to the metabolic reactions that are explicitly specified in the considered network, we assume that some metabolites are used in subsequent stages of the metabolism. Therefore, we will include consumption rates in the model for those reactions in the form $X_{s} \rightarrow \ldots$ which will be denoted $\varphi_{s 0}\left(x_{s}\right)$.

Because it is natural to think that the larger the concentration of $X_{s}$ is, the faster the reaction will take place, and the larger the concentration of any given inhibitor is, the slower the reaction will take place, we impose the following assumption for the reaction rates:

Assumption 1. For all $s, p$ such that the reaction $X_{s} \rightarrow X_{p}$ belongs to the metabolic network, the function

$$
\varphi_{s p}\left(x_{s}, x^{[p]}\right)
$$

is locally Lipschitz on $\mathbb{R}_{+} \times \mathbb{R}_{+}^{n_{p}}$, satisfies $\varphi_{s p}\left(0, x^{[p]}\right)=0$, is increasing in $x_{s}$ for $x_{s} \geq 0$ and decreasing in $x_{j}^{[p]}$ for $x_{j}^{[p]} \geq 0$ (and $x_{s}>0$ ).

We represent the network under the form of a graph; we therefore need the following definition from graph theory to precisely define the class of metabolic networks that we consider: 
Definition 1. A directed graph is called an arborescence if, from a given node $x$, known as the root node, there is exactly one elementary path from this node to any other node $y$.

This definition leads to the following assumption that we impose on the metabolic networks that we consider

Assumption 2. a): the involved species are denoted $X_{1}, X_{2}, \cdots, X_{n}$;

b): the graphic representation of the network (with the different metabolites as nodes and the different reactions as oriented edges) is an arborescence with $X_{1}$ as root;

c): the only inhibited reactions are the reactions descending from the root $X_{1} \rightarrow$ $X_{p}$; they are inhibited by metabolites from the (sub)-arborescence rooted in $X_{p}$

Stemming from the definition and known properties of arborescences, Assumption $2 \mathrm{~b}$ has the following consequence on the class of metabolic networks that we consider:

(i): Each metabolite is produced by a single other metabolite;

(ii): There is no cycle of reactions;

Remark that the conditions of Assumption 2 are satisfied by the networks of Figure 1, but they are also satisfied by more complex networks, as illustrated on Figure 3 .

With these definitions and notations, we shall now define a mass-balance dynamical model in the form

$$
\dot{x}=\Phi(x)-\mu x+c e_{1}
$$

where $x=\left(x_{1}, \cdots, x_{n}\right)^{T} \in \mathbb{R}_{+}^{n}$, and $x_{i}$ denotes the molar fraction of the metabolite $X_{i}$ inside the cell. The factor $\mu \geq 0$ represents the specific growth rate of the cell: we assume that the cell metabolism is analyzed during a period of exponential cell growth with a constant specific growth rate $\mu$. The vector $e_{1}=(1,0, \cdots, 0)^{T}$ and the scalar $c$ denote the constant supply rate of the metabolite $X_{1}$ at the root of the network. The function $\Phi$ includes all the reaction rates.

In order to specify $\Phi(x)$, we introduce the following notations:

Notation. $\quad-\mathcal{P}(j)=\left\{k \mid\right.$ the reaction $X_{j} \rightarrow X_{k}$ belongs to the network $\} \cdot \mathcal{P}(j)$ defines the set of all metabolites that are produced by reactions having $X_{j}$ as substrate. If there is a consumption term in the form $\varphi_{j 0}\left(x_{j}\right)$ in the derivative of $x_{j}$, the index 0 is included in $\mathcal{P}(j)$.

- $\mathcal{A}(j)=\left\{k \mid X_{k}\right.$ belongs to the arborescence with its root in $\left.X_{j}\right\}$. " 0 " is not included in $\mathcal{A}(j)$.

It can easily be seen that $\mathcal{P}(j) \backslash\{0\}$ is a subset of $\mathcal{A}(j)$, and that for all $k \notin \mathcal{P}(1)$, $x^{[k]}=\emptyset$ because of Assumption 2 .

From the arborescence structure, it is clear that we can separate the metabolites into three different families:

- the root $X_{1}$ : since there is a constant supply rate $c$ of $X_{1}$, the corresponding mass-balance equation is the following:

$$
\dot{x}_{1}=c-\sum_{k \in \mathcal{P}(1)} \varphi_{1 k}\left(x_{1}, x^{[k]}\right)-\mu x_{1}
$$




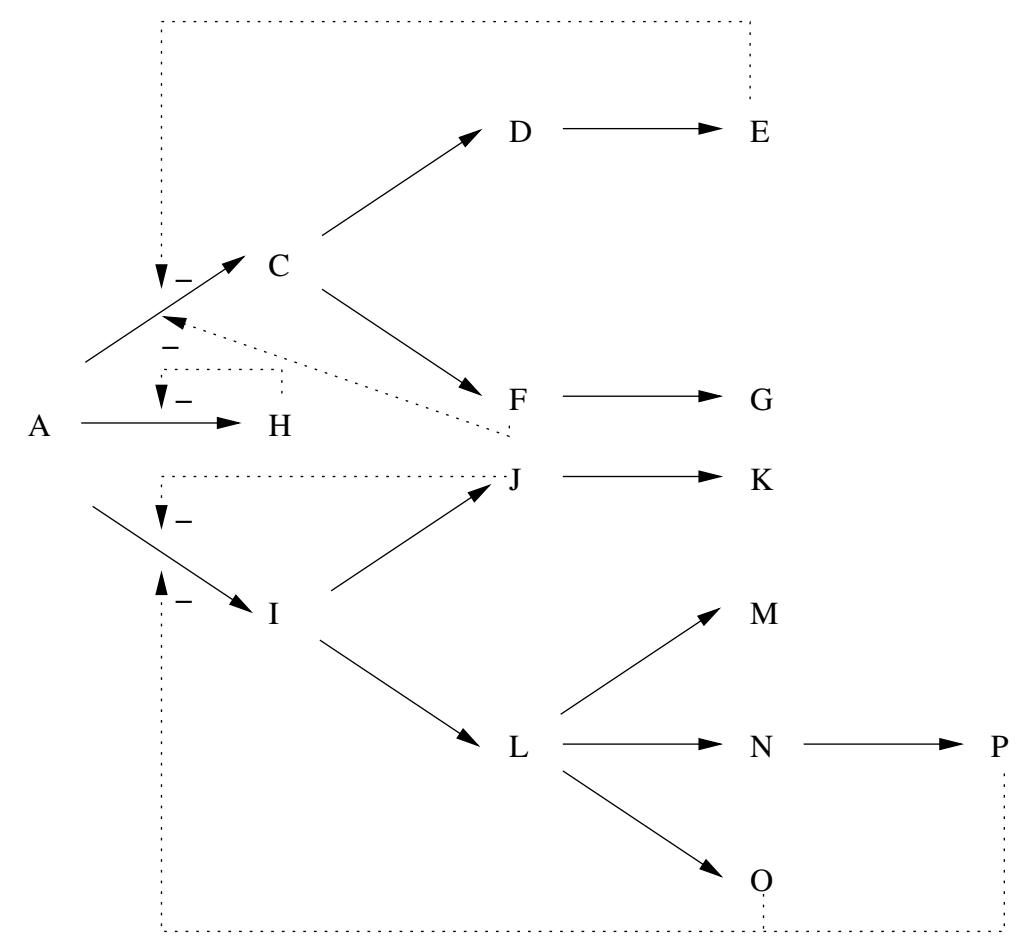

FIGURE 3. Example of a network satisfying Assumption 2, In this network, it is seen, for example, that the reaction $A \rightarrow I$ is inhibited by the metabolites $J, O$ and $P$; these simultaneous inhibiting actions can be either the result of cumulative inhibition of a single enzyme that catalyzes $A \rightarrow I$ by all the metabolites or the parallel inhibition of several isofunctional enzymes that catalyze $A \rightarrow I$.

- the intermediate metabolite $X_{j}$, which is the result of the reaction $X_{i} \rightarrow X_{j}$ :

$$
\dot{x}_{j}=\varphi_{i j}\left(x_{i}, x^{[j]}\right)-\sum_{k \in \mathcal{P}(j)} \varphi_{j k}\left(x_{j}\right)-\mu x_{j}
$$

- the boundary metabolite $X_{j}$ ( $\operatorname{such}$ that $\mathcal{P}(j)=\{0\}$ or $\emptyset$ ), which is the product of a reaction $X_{i} \rightarrow X_{j}$ :

$$
\dot{x}_{j}=\varphi_{i j}\left(x_{i}, x^{[j]}\right)-\varphi_{j 0}\left(x_{j}\right)-\mu x_{j}
$$

Under Assumption 2, we can only have $x^{[j]}=x_{j}$ (only if $i=1$ ) or $x^{[j]}=\emptyset$.

The analysis of system (1)-(2)-(3) is an extension of what was previously known. Indeed, [18, [9, [13], and [3] proved stability of the equilibrium or the existence of a limit-cycle for various metabolic/genetic networks. They all handled the case where the reaction network is a single chain of reactions with inhibition induced on the reactions by the last metabolite only; in the case of [18] and [9], this inhibition only acts on the production of the first metabolite. Our proof of uniqueness of the equilibrium goes beyond those classes as it applies to (1)-(2)-(3) and, though we will restrict our exposed proof of stability to a chain of reactions for the sake of clarity, our stability result is also valid for (1)-(2)-(3) (see 8 for more details). A final difference is that, in the classical literature, the metabolic network is rooted in 
an unmodeled metabolite whose concentration is supposed to stay constant, while we consider a different situation where the network is rooted in a metabolite which is brought into the system at a constant supply rate (the concentration of this metabolite therefore needs to be modeled: it is $X_{1}$ ).

3. Equilibrium of a metabolic network. The first step in our analysis consists in the search of equilibria for the model. Based on equations (1)-(2)-(3), we can compute the dynamics of the total mass $\sum_{l=1}^{n} x_{l}$ of the system:

$$
\frac{d}{d t}\left(\sum_{l=1}^{n} x_{l}\right)=c-\mu \sum_{l=1}^{n} x_{l}-\sum_{\{k \mid 0 \in \mathcal{P}(k)\}} \varphi_{k 0}\left(x_{k}\right)
$$

and of the total mass of the subnetwork corresponding to the arborescence which is rooted in $X_{j}$

$$
\frac{d}{d t}\left(\sum_{l \in \mathcal{A}(j)} x_{l}\right)=\varphi_{i j}\left(x_{i}, x^{[j]}\right)-\mu \sum_{l \in \mathcal{A}(j)} x_{l}-\sum_{\{k \mid 0 \in \mathcal{P}(k) \text { and } k \in \mathcal{A}(j)\}} \varphi_{k 0}\left(x_{k}\right)
$$

These expressions will be critical in the proof of the following proposition:

Proposition 1. If Assumptions 1 and 2 are satisfied, then:

(A): the system (1)-(2)-(3) is positive;

(B): if $\mu=0$ and all $\varphi_{s, p}\left(x_{s}, x^{[p]}\right)$ (where $p$ can be 0 ) are increasing in $x_{s}$ then there is at most one equilibrium $\bar{x}=\left(\bar{x}_{1}, \cdots, \bar{x}_{n}\right)$ of (1)-(2)-(3) in $\mathbb{R}_{+}^{n}$;

(C): if $\mu>0$, then system (1)-(2)-(3) has a unique equilibrium $\bar{x}=\left(\bar{x}_{1}, \cdots, \bar{x}_{n}\right)$ in $\mathbb{R}_{+}^{n}$. Moreover, the solutions of (1)-(2)-(3) are bounded for any initial condition in $\mathbb{R}_{+}^{n}$.

Proof. (A) is easily seen by considering the system on the boundaries of the positive orthant.

The proofs of (B) and (C) are very similar. We write the proof for (B) and highlight the differences that arise for the proof of $(\mathrm{C})$.

We will first consider system (2)-(商) with $x_{1}=\bar{x}_{1}$ as constant input. For any value of $\bar{x}_{1}$, we will denote by $\left(\bar{x}_{2}, \cdots, \bar{x}_{n}\right)$ the equilibrium of $(2)$-(3); this equilibrium is a function of $\bar{x}_{1}$, so that we will state that it is $\left(\bar{x}_{2}, \cdots, \bar{x}_{n}\right)\left(\bar{x}_{1}\right)$. We will now show, by induction, that every element $\bar{x}_{i}$ is an increasing function of $\bar{x}_{1}$ (resp. non-decreasing in case $(\mathrm{C})$ ).

The initial step of the proof considers the equilibrium of (3), an equation characterizing the evolution of the concentration of a boundary metabolite, with $x_{i}=\bar{x}_{i}$ as constant input

$$
\varphi_{i j}\left(\bar{x}_{i}, \bar{x}^{[j]}\right)-\varphi_{j 0}\left(\bar{x}_{j}\right)-\mu \bar{x}_{j}=0
$$

where $\bar{x}^{[j]}=\bar{x}_{j}$ or $x^{[j]}=\emptyset$. When $\bar{x}_{i}=0, \bar{x}_{j}=0$ is the only solution. Also, the left-hand side of this equation is an increasing function of $\bar{x}_{i}$ (resp. non-decreasing in case $(\mathrm{C})$ ) and a decreasing function of $\bar{x}_{j}$. It is then easily seen that, if we increase $\bar{x}_{i}, \bar{x}_{j}$ needs also to be increased (resp. increased or kept constant) to keep this equality satisfied. We then have that, in this case, $\bar{x}_{j}\left(\bar{x}_{i}\right)$ is an increasing function such that $\bar{x}_{j}(0)=0$ (resp. non-decreasing function such that $\left.\bar{x}_{j}(0)=0\right)$. When $\mu=0$ (which can only happen in case $(\mathrm{B})$ ), the definition of $\bar{x}_{j}($.$) could$ be limited to an interval $\left[0, \bar{x}_{i}^{m}\right)$ with $\lim _{\bar{x}_{i} \rightarrow \bar{x}_{i}^{m}} \bar{x}_{j}\left(\bar{x}_{i}\right)=+\infty$. Indeed, in the case where $\varphi_{j 0}\left(x_{j}\right)<B_{j}$ for all $x_{j} \geq 0$ and for some $B_{j}>0$, there might exist some $\bar{x}_{i}^{m}$ such that $\varphi\left(\bar{x}_{i}, \bar{x}_{j}\right)>B_{j}$ for all $\bar{x}_{i}>\bar{x}_{i}^{m}$ and all $\bar{x}_{j} \geq 0$. 
Let us now make the following induction hypothesis: for a given $j$, the functions $\bar{x}_{k}\left(\bar{x}_{j}\right)$ are increasing (resp. non-decreasing) functions for all $k \in \mathcal{A}(j)$ with $\bar{x}_{k}(0)=$ 0 . We then study the equilibrium of the mass-balance of the arborescence that has its root in $X_{j}$. From (5):

$$
\varphi_{i j}\left(\bar{x}_{i}, \bar{x}^{[j]}\left(\bar{x}_{j}\right)\right)-\mu \sum_{l \in \mathcal{A}(j)} \bar{x}_{l}\left(\bar{x}_{j}\right)-\sum_{\{k \mid 0 \in \mathcal{P}(k) \text { and } k \in \mathcal{A}(j)\}} \varphi_{k 0}\left(\bar{x}_{k}\left(\bar{x}_{j}\right)\right)=0
$$

With a similar argument to that of the initial step, we see that $\bar{x}_{j}$ is an increasing (resp. non-decreasing) function of $\bar{x}_{i}$ and that $\bar{x}_{j}(0)=0$. The same can be said for all $\bar{x}_{k}$ with $k \in \mathcal{A}_{j}$ because they are already increasing (resp. non-decreasing) functions of $x_{j}$.

By induction, we then have that every $\bar{x}_{k}\left(\bar{x}_{1}\right)$ is an increasing function of $\bar{x}_{1}$ defined on the interval $\left[0, \bar{x}_{1}^{m}\right.$ ) (resp. non decreasing function defined for all $\bar{x}_{1} \geq 0$ ). An equilibrium of the whole system then has to satisfy the equilibrium of the total mass-balance. From (4), this comes to:

$$
\mu \sum_{l=1}^{n} \bar{x}_{l}\left(\bar{x}_{1}\right)+\sum_{\{k \mid 0 \in \mathcal{P}(k)\}} \varphi_{k 0}\left(\bar{x}_{k}\left(\bar{x}_{1}\right)\right)=c
$$

The system admits as many equilibria as this equation has roots. In case (B), the left-hand side is increasing from 0 when $\bar{x}_{1}$ increases from 0 to $\bar{x}_{1}^{m}$ (because of the second term). Therefore, if there exists an equilibrium, it is unique. In case (C), the left-hand side is increasing from 0 to $+\infty$ when $\bar{x}_{1}$ increases from 0 to $+\infty$ (because of the $\mu \bar{x}_{1}$ term), so that the equilibrium exists and it is unique.

The final point of (C) is a direct consequence of (4); this implies

$$
\frac{d}{d t}\left(\sum_{l=1}^{n} x_{l}\right) \leq c-\mu \sum_{l=1}^{n} x_{l}
$$

which clearly implies boundedness of the solutions when $\mu>0$.

We have shown in 8 that this result is even valid for a larger class of metabolic networks, namely networks that satisfy a weaker version of Assumption 2 : the reactions $X_{s} \rightarrow X_{p}$ are inhibited by metabolites from the (sub)-arborescence rooted in $X_{p}$. Compared to the case where Assumption $2 \mathrm{c}$ is satisfied, inhibiting metabolites are not restricted to act on reactions that have $X_{1}$ as substrate, but can act on any reactions that are upstream in their own pathway (like in the amino-acids pathways of Figure 2). Note that the uniqueness of this equilibrium is in agreement with the results of [14, 7], and [16, which state that at least a positive feedback loop is necessary in the system in order to have multiple equilibria; the absence of positive feedback loop can be shown in our model.

Uniqueness of the equilibrium, especially when it is coupled with boundedness of solutions, gives hope of having some general result about the structural global asymptotic stability of the equilibrium. In the next section, we will impose constraints on the rates so that we will be able to prove global attractivity of the single equilibrium.

4. Stability analysis. In this section, we will study the stability analysis of the class of metabolic networks that was described in Section 2.

In the main part of this section, we will present a very detailed stability analysis for a rather simple metabolic network, ending up with a sufficient condition for 
global asymptotic stability of the equilibrium point. We do so in order to emphasize on our techniques without hiding them behind too many computations. Then, in a second part, we will only provide a similar sufficient condition corresponding to the general metabolic network described in Section 2.

We consider as a toy model for our stability analysis, a metabolic network modeling a sequence of mono-molecular enzyme-catalyzed reactions where $X_{i} \quad(i=$ $1, \cdots, n)$ represent the successive metabolites of the pathway:

$$
X_{1} \rightarrow X_{2} \rightarrow \cdots \rightarrow X_{n} .
$$

We assume that there is only one sequential feedback inhibition (cf. 17]), i.e., the last metabolite $X_{n}$ acts as an inhibitor of the first reaction $X_{1} \longrightarrow X_{2}$. Moreover, to make the computations less painful to follow, we also made a more explicit choice for the functions $\varphi_{s p}\left(x_{s}, x^{[p]}\right)$. More precisely, the velocity of each enzymatic reaction $X_{i} \longrightarrow X_{i+1}$ is represented by a Michaelis-Menten kinetic function :

$$
\varphi_{i}\left(x_{i}\right)=\frac{a_{i} x_{i}}{k_{i}+x_{i}},
$$

where $x_{i}$ denotes the intracellular molar fraction of the metabolite $X_{i}, a_{i}$ is the maximal velocity and $k_{i}$ the so-called half-saturation constant. It is assumed that the velocity of the first reaction $X_{1} \longrightarrow X_{2}$ is inhibited by the last metabolite with a multiplicative hyperbolic inhibition function of the form:

$$
\psi_{\alpha}\left(x_{n}\right)=\frac{1}{1+\alpha x_{n}}
$$

Under these assumptions and notations, the mass balance dynamical model under study in this section is formulated as:

$$
\left\{\begin{array}{l}
\dot{x}_{1}=-\frac{a_{1} x_{1}}{\left(k_{1}+x_{1}\right)\left(1+\alpha x_{n}\right)}-\mu x_{1}+c, \\
\dot{x}_{2}=\frac{a_{1} x_{1}}{\left(k_{1}+x_{1}\left(1+\alpha x_{n}\right)\right.}-\frac{a_{2} x_{2}}{\left(k_{2}+x_{2}\right)}-\mu x_{2}, \\
\dot{x}_{i}=\frac{a_{i-1} x_{i-1}}{\left(k_{i-1}+x_{i-1}\right)}-\frac{a_{i} x_{i}}{\left(k_{i}+x_{i}\right)}-\mu x_{i}, 3 \leq i \leq n,
\end{array}\right.
$$

where $n \geq 3$ is a positive integer, $x=\left(x_{1}, \cdots, x_{n}\right)^{T} \in \mathbb{R}^{n}$, and all the $a_{i}, k_{i}$ 's, $c, \alpha, \mu$ are positive constants.

Note that, without inhibition (i.e. $\alpha=0$ ), system (8) is clearly compartmental and cooperative, which implies that it has a single globally asymptotically stable equilibrium. But if there is inhibition, the system is no longer cooperative and the stability analysis is more difficult.

\subsection{Notations and statement of the theorem.}

4.1.1. Notations. Consider the metabolic system with feedback inhibition (8). Up to a change of variable (the $x_{i}$ 's are multiplied by $\alpha$ ) and a time reparameterization (the time is multiplied by $\mu$ ), we may assume that $\mu=\alpha=1$ and the model is rewritten:

$$
(\Sigma)\left\{\begin{array}{l}
\dot{x}_{1}=-\varphi_{1}\left(x_{1}\right) \psi\left(x_{n}\right)-x_{1}+c, \\
\dot{x}_{2}=\varphi_{1}\left(x_{1}\right) \psi\left(x_{n}\right)-\varphi_{2}\left(x_{2}\right)-x_{2}, \\
\dot{x}_{i}=\varphi_{i-1}\left(x_{i-1}\right)-\varphi_{i}\left(x_{i}\right)-x_{i}, 3 \leq i \leq n,
\end{array}\right.
$$

where $\varphi_{i}$ is defined in (6) and $\psi:=\psi_{1}$ in (7). In this model, $c$ stands for $\frac{\alpha c}{\mu}$ (with the original $c$ in that last formula) and similarly, for $1 \leq i \leq n, a_{i}$ stands for $\frac{\alpha a_{i}}{\mu}$ and $k_{i}$ for $\alpha k_{i}$.

We introduce some notations: for $1 \leq i \leq n, F_{i}(x)$ is the real function defining $\dot{x}_{i}$. For $2 \leq i \leq n, f_{i}(x)=x+\varphi_{i}(x)$ and $f_{1, x_{n}}(x)=x+\psi\left(x_{n}\right) \varphi_{1}(x)$. It is clear that 
the $\varphi_{i}$ 's, $1 \leq i \leq n$, are monotone functions on $\mathbb{R}_{+}$and realize bijections between $\mathbb{R}_{+}$and $\left[0, a_{i}\right)$. We use $\varphi_{i}^{-1}$ to denote the inverse function. For $2 \leq i \leq n$, the $f_{i}$ 's are monotone functions on $\mathbb{R}_{+}$and realize bijections from $\mathbb{R}_{+}$to $\mathbb{R}_{+} ; f_{i}^{-1}$ denotes the inverse function of $f_{i}$ and $g_{i}=\varphi_{i} \circ f_{i}^{-1}$. Let $M: \mathbb{R}_{+} \rightarrow \mathbb{R}_{+}$by $M=f_{n}^{-1} \circ g_{n-1} \circ \cdots \circ g_{2}$.

For every $x \geq 0$ and $2 \leq i \leq n$, we have

$$
1<f_{i}^{\prime}(x)=1+\varphi_{i}^{\prime}(x) \leq 1+\frac{a_{i}}{k_{i}}, \frac{k_{i}}{a_{i}+k_{i}} \leq\left(f_{i}^{-1}\right)^{\prime}(x)=\frac{1}{1+\varphi_{i}^{\prime}\left(f_{i}^{-1}(x)\right)}<1 .
$$

Note that the $f_{i}$ 's, the $\varphi_{i}$ 's are concave functions on $\mathbb{R}_{+}$(negative second derivative) for $2 \leq i \leq n$. This implies that the $g_{i}$ 's are also concave. Therefore, we have for $2 \leq i \leq n$ and every $x \geq 0$

$$
0<g_{i}^{\prime}(x)=\frac{\varphi_{i}^{\prime}\left(f_{i}^{-1}(x)\right)}{1+\varphi_{i}^{\prime}\left(f_{i}^{-1}(x)\right)} \leq g_{i}^{\prime}(0)=\frac{a_{i}}{k_{i}+a_{i}},
$$

and since

$$
M^{\prime}(x)=\left(f_{n}^{-1}\right)^{\prime}\left(g_{n-1} \circ \cdots \circ g_{2}(x)\right)\left[\prod_{i=3}^{n-1} g_{i}^{\prime}\left(g_{i-1} \circ \cdots \circ g_{2}(x)\right)\right] g_{2}^{\prime}(x),
$$

we can then conclude from (10) and (11) that, for every $x \geq 0$

$$
0<M^{\prime}(x)<\prod_{i=2}^{n-1} \frac{a_{i}}{k_{i}+a_{i}} .
$$

As for $f_{1, x_{n}}$, for every $x_{n} \in \mathbb{R}_{+}$, it behaves like any $f_{i}, 2 \leq i \leq n$. Define $z$ : $\mathbb{R}_{+} \rightarrow[0, c)$ by $z(b)=f_{1, b}^{-1}(c)$. Later we will study in more details that application. Let $F$ be the vector field on $\mathbb{R}^{n}$ simply defined by the right-hand side of $(\Sigma)$. Let $K=\mathbb{R}_{+}^{n}$ the non-negative orthant and $K_{+}$the positive orthant. The positive cone $K$ defines a closed partial order relation $\leq$ on $\mathbb{R}^{n}$ defined by $x \leq y$ if and only if $y-x \in K$. It means that $x_{i} \leq y_{i}$ holds for every $1 \leq i \leq n$. We write $x<y$ if $x \leq y$ and $x \neq y$, and $x \ll y$ whenever $y-x \in \operatorname{Int}(K)=K_{+}$. This notation extends trivially to subsets of $\mathbb{R}^{n}$.

If $f: \mathbb{R}_{+} \rightarrow \mathbb{R}$, set $\varlimsup \lim f=\limsup _{t \rightarrow \infty} f$ and $\underline{\lim f} f=\liminf _{t \rightarrow \infty} f$. This notation is naturally extended to the vectorial case using the partial order defined previously. We will also consider sometimes the function $V: \mathbb{R}^{n} \rightarrow \mathbb{R}$ defined by $V(x)=\sum_{i=1}^{n} x_{i}$. An $m \times m$ matrix $A=\left(a_{i j}\right)$ is said to be irreducible if for every nonempty, proper subset $I \subset\{1, \cdots, n\}$, there is an $i \in I$ and $j \in\{1, \cdots, n\} / I$ such that $a_{i j} \neq 0$. There is a graph-theoretic formulation of irreducibility (cf. [15]): consider the directed graph $\mathcal{G}$ whose set of vertices is $\{1, \cdots, n\}$; two vertices $i, j$ have a directed edge from $i$ to $j$ if $a_{i j} \neq 0$. Then $A$ is irreducible if its directed graph $\mathcal{G}$ is connected.

A dynamical system $(G)$ given by $\dot{x}=G(x), x \in D$ with $D$ open, $G: D \rightarrow \mathbb{R}^{n}$ of class $C^{1}$ is said to be cooperative (see [15]) if, for every $x \in D, 1 \leq i, j \leq n$ and $i \neq j$,

$$
\frac{\partial G_{i}}{\partial x_{j}} \geq 0
$$

If, in addition, the Jacobian matrix $D G(x)$ is irreducible for every $x \in D$, then $(G)$ is said to be irreducible cooperative. 
Remark 1. It is worth noticing that $(\Sigma)$ is not cooperative with respect to the partial order defined by $K$ or by any other partial order defined by an orthant of $\mathbb{R}^{n}$ (cf. [15]). Even though the Jacobian matrix $D F(x)$ is sign-stable and signsymmetric (if $n \geq 4$ ), it fails the test for the existence of $\beta=\left(\beta_{1}, \cdots, \beta_{n}\right) \in\{0,1\}^{n}$ so that, for every $i \neq j, \beta_{i}+\beta_{j}=s_{i j}(\bmod 2)$, where

$$
s_{i j}=\left\{\begin{array}{l}
0, \text { if } \frac{\partial f_{i}}{\partial x_{j}}+\frac{\partial f_{j}}{\partial x_{i}}>0, \\
1, \text { if } \frac{\partial f_{i}}{\partial x_{j}}+\frac{\partial f_{j}}{\partial x_{i}}<0, \\
\in\{0,1\}, \text { if } \frac{\partial f_{i}}{\partial x_{j}}+\frac{\partial f_{j}}{\partial x_{i}} \equiv 0 .
\end{array}\right.
$$

Indeed, we have $\beta_{1}+\beta_{n}=0, \beta_{1}+\beta_{2}=0$ and $\beta_{2}+\beta_{n}=1$. Adding the two last equations implies that $\beta_{1}+\beta_{n}=1$, contradicting the first equation. It should be pointed out that the coefficient in the Jacobian $D F(x)$ that renders $(\Sigma)$ non cooperative is $\frac{\partial f_{2}}{\partial x_{n}}$, which is indeed negative.

We will consider auxiliary systems $(\Sigma)_{b}, b \geq 0$, given by

$$
(\Sigma)_{b}\left\{\begin{array}{l}
\dot{x}_{1}=-\varphi_{1}\left(x_{1}\right) \psi\left(x_{n}\right)-x_{1}+c, \\
\dot{x}_{2}=\varphi_{1}\left(x_{1}\right) \psi(b)-\varphi_{2}\left(x_{2}\right)-x_{2}, \\
\dot{x}_{k}=\varphi_{k-1}\left(x_{k-1}\right)-\varphi_{k}\left(x_{k}\right)-x_{k}, 3 \leq k \leq n,
\end{array}\right.
$$

where the difference with $(\Sigma)$ lies in the equation defining $\dot{x}_{2}$ : the variable $x_{n}$ is frozen at the constant value $b$. We use $F_{b}(x)$ to denote the right-hand side of $(\Sigma)_{b}$. Now, the $(2, n)$-coefficient in $D F_{b}(x)$ is identically equal to zero. If $x \in K$, we use $\gamma_{x}, \gamma_{x}^{b}$ respectively, to denote the trajectory of $(\Sigma),(\Sigma)_{b}$ respectively, which starts at $x$.

Remark 2. At the light of Proposition 1, the relevance of the auxiliary systems $(\Sigma)_{b}$ for understanding the dynamics of $(\Sigma)$ can be put forward. It is based on the two following remarks:

$(a)$ : for every $b \geq 0,(\Sigma)_{b}$ is an irreducible cooperative system (use the graphtheoretic formulation of irreducibility). This easily implies that $(\Sigma)_{b}$ verifies (2) (cf. Theorem 1.1 p.56 of [15]) and (3) (with possibly another positive constant instead of $c)$. In fact $(\Sigma)_{b}$ is a hypercycle for which a Poincaré-Bendixson theory was developed for the compact $\omega$-limit sets of $(\Sigma)_{b}$, regardless of the dimension of the system (cf. 12). Then, we expect taking advantage of the many deep results relative to that class of irreducible cooperative systems (cf. [12] and [15]).

(b): for every $x \in K$ and $0 \leq b_{0}<b_{1}$, we have

$$
b_{0} \leq x_{n} \leq b_{1} \Rightarrow F_{b_{1}}(x) \leq F(x) \leq F_{b_{0}}(x),
$$

and, if $x_{1}>0$, then $\leq$ can be replaced everywhere by $<$ in the above equation. The monotonicity property expressed in (15) translates to the trajectories of $F$ and $F_{b}$ as explained next. Assume that we have shown the existence of $0 \leq b_{0}<b_{1}$ such that for every $x \in K$, there is some $t_{x}>0$ for which

$$
b_{0} \leq x_{n}(t) \leq b_{1} \text {, if } t \geq t_{x} .
$$

(This is clearly the case by $(A)$ of Proposition 11) Using (15), we have, for $t \geq t_{x}$

$$
F_{b_{1}}\left(\gamma_{x}(t)\right) \leq F\left(\gamma_{x}(t)\right) \leq F_{b_{0}}\left(\gamma_{x}(t)\right)
$$


Set $y_{x}=\gamma_{x}\left(t_{x}\right)$. Since $F_{b}$ is a function of type $\mathcal{K}$, we can apply a standard theorem of comparison for differential inequalities (cf. for instance Theorem 10 p.29 of [5]): for $t \geq t_{x}$,

$$
\gamma_{y_{x}}^{b_{1}}\left(t-t_{x}\right) \leq \gamma_{x}(t) \leq \gamma_{y_{x}}^{b_{0}}\left(t-t_{x}\right)
$$

Assume now that, according to Part $(a)$, the $\omega$-limit sets of $(\Sigma)_{b_{0}}$ and $(\Sigma)_{b_{1}}$ are investigated in details and one is able to show e.g. that every trajectory of $(\Sigma)_{b_{0}}$ $\left((\Sigma)_{b_{1}}\right.$ respectively) starting in $K$ converges to $x^{b_{0}}\left(x^{b_{1}}\right.$ respectively). In addition, assume that $b_{0} \ll x^{b_{0}}$ and $x^{b_{1}} \ll b_{1}$. Then the pair $\left(x_{n}^{b_{0}}, x_{n}^{b_{1}}\right)$ can be used in (16) instead of $\left(b_{0}, b_{1}\right)$ in the bounding process for $\gamma_{x}(t)$ described above. If that procedure can be reproduced, one may hope to get more and more precise information on the $\omega$-limit sets of $(\Sigma)$. It is even tempting to conjecture that every trajectory of $(\Sigma)$ starting in $K$ converges to $\bar{x}$. We prove it but for a restricted set of the problem's parameters.

Theorem 1. Under the following condition $(H)$,

$$
(H)\left(a_{1}+c\right) \prod_{i=2}^{n-1} \frac{a_{i}}{k_{i}+a_{i}} \leq 1
$$

the system $(\Sigma)$ is globally asymptotically stable in $K$ with respect to $\bar{x}$.

Remark 3. We may express condition $(H)$ in terms of the original parameters, i.e. with $\alpha$ and $\mu$ (for system (8)). Equation (18) becomes

$$
\frac{\alpha}{\mu}\left(a_{1}+c\right) \prod_{i=2}^{n-1} \frac{a_{i}}{\mu k_{i}+a_{i}} \leq 1 .
$$

It is not surprising that if $\alpha=0$ (i.e. no inhibition) or if $\mu$ is large enough (fast growth of the cell) then the condition expressed in (19) holds true. Indeed, in the first case, the system is cooperative, so that the equilibrium is globally asymptotically stable; in the second case, the consumption of each metabolite through the metabolic reactions is dominated by their dilution, so that system (8) is close to a simple dilution process.

Remark 4. The theory recently developed by Angeli and Sontag about interconnections of controlled monotone systems, see ([2]), applies to the system studied in that paper. Both approaches rely on the same idea namely the monotone behavior of the system with respect particular (cluster of) coordinates. More precisely, one can write the variable $x=\left(x_{1}, \cdot, x_{n}\right)$ as the pairing $(y, u)$ where the system has two different monotonicity behaviors with respect to $y$ and with respect to $u$. The beautiful idea of [2] is to look at $u$ as a control variable and to consider the global system as the feedback interconnection of two control systems, the first one with $u$ as the control variable and the second one as $y$ as control variable. Our approach is more elementary in the sense that it does not borrow concepts from control theory but it is more general because it requires less assumptions to be applied. Indeed, the key technical object of [2] is the Input/State characteristic map, and for it to be defined, one must impose restrictions on the asymptotic behavior of appropriate sub-systems. Note, however, that the necessary conditions derived in the present paper, for uniqueness of a fixed point and convergence to it, can be recovered by the methods of [2], which furnish in the case under study exactly the same results as ours. 


\subsection{Proof of Theorem 1 .}

4.2.1. Technical lemmas. Before starting the proof of the theorem, we establish a series of lemmas which will be useful for the analysis of system $\left(\Sigma_{b}\right)$. We first study the application $z: \mathbb{R}_{+} \rightarrow[0, c)$ by $z(b)=f_{1, b}^{-1}(c)$. We have

Lemma 1. (i): The application $z$ is strictly increasing from $\mathbb{R}_{+}$to $[0, c)$;

(ii): The application $\varphi_{1} \circ z$ is strictly increasing from $\mathbb{R}_{+}$to $\left[0, \varphi_{1}(c)\right.$ ) and is concave.

Proof of Lemma 1: The expression of $z(y)$ comes from the equation $\dot{x}_{1}=0$. Differentiating this equation with respect to $y$ results in an expression $z^{\prime}(y)=$ $l\left(z(y), \frac{1}{1+y}\right)$ for some function $l$. We then isolate $\frac{1}{1+y}$ in the equation $\dot{x}_{1}=0$ and substitute the resulting expression into $l$, so that

$$
z^{\prime}(y)=\frac{(c-z(y))^{2}\left(z(y)+k_{1}\right)^{2}}{a_{1}\left(z(y)^{2}+c k_{1}\right)}
$$

and $z^{\prime}(y)>0$ for all $y \geq 0$ because $z(y)<c$ for all $y \geq 0$. Item $(i)$ is proved.

The expressions of $\frac{d \varphi(z(y))}{d y}$ and $\frac{d^{2} \varphi(z(y))}{d y^{2}}$ result from straightforward computations:

$$
\begin{gathered}
\frac{d \varphi(z(y))}{d y}=\varphi^{\prime}(z) z^{\prime}(y)=\frac{k_{1}(c-z(y))^{2}}{z(y)^{2}+c k_{1}} \\
\frac{d^{2} \varphi(z(y))}{d y^{2}}=\frac{d}{d z}\left(\frac{d \varphi(z(y))}{d y}\right) z^{\prime}(y)=-\frac{2 k_{1} c(c-z(y))^{3}\left(z(y)+k_{1}\right)^{3}}{\left(z(y)^{2}+c k_{1}\right)^{3}}
\end{gathered}
$$

so that $\frac{d \varphi(z(y))}{d y}>0$ and $\frac{d^{2} \varphi(z(y))}{d y^{2}}<0$ because $z(y)<c$.

Next, we determine, for $b \in[0, c]$, the equilibrium set $E_{b}$ of $(\Sigma)_{b}$, i.e. the set of the equilibrium points of $(\Sigma)_{b}$.

Lemma 2. A point $e \in K$ is an element of $E_{b}$ if and only if its $n$-th coordinate $e_{n}$ is solution in $\mathbb{R}_{+}$of the following equation in the unknown $y$

$$
y=M\left(\varphi_{1}(z(y)) \psi(b)\right) .
$$

Moreover the previous equation always has solutions and it has exactly one if condition $(H)$ holds.

Remark 5. We chose in this paper to investigate the sets $E_{b}$ 's in an elementary way rather than using the deep work of [1] and [12. Doing so leads to results on $E_{b}$ which are only valid under condition $(H)$, even though they should hold in a more general setting.

Proof of Lemma 2: if $e \in E_{b}$, then $e_{n}=M\left(\varphi_{1}\left(e_{1}\right) \psi(b)\right)$. Then (23) follows by definition of $z$. When considering the solutions of (23), we are looking for the zeros of the function $h_{b}: \mathbb{R}_{+} \rightarrow \mathbb{R}$, defined by

$$
h_{b}(y)=y-M\left(\varphi_{1}(z(y)) \psi(b)\right) .
$$

Note that $h_{b}(0)<0$ and $h_{b}$ tends to $+\infty$ if $y$ tends to $+\infty$ (indeed, $\varphi_{1}(z(y)) \psi(b)$ is bounded). Therefore, if $h_{b}$ is strictly increasing, then $E_{b}$ will have a unique equilibrium point. We show next that this holds true under condition $(H)$.

The derivative of $h_{b}$ is

$$
h_{b}^{\prime}(y)=1-M^{\prime}\left(\varphi_{1}(z(y)) \psi(b)\right) \frac{d \varphi(z(y))}{d y} \psi(b),
$$


Equation (12) gives an upper bound on $M^{\prime}$ and concavity of $\varphi(z(y))$ implies that

$$
\frac{d \varphi(z(y))}{d y}(y) \psi(b) \leq \frac{d \varphi(z(y))}{d y}(0) \psi(b) .
$$

We also see from $(\overline{20})$ and $(\overline{22})$ that $\frac{d}{d z}\left(\frac{d \varphi(z(y))}{d y}\right)<0$ so that

$$
\left.\frac{d \varphi(z(y))}{d y} \underset{\mid z=z(0)}{<\frac{d \varphi(z(y))}{d y}}\right|_{\mid z=0}=c .
$$

We then have a unique zero to $h_{b}$ if

$$
1-\prod_{i=2}^{n-1} \frac{a_{i}}{k_{i}+a_{i}} c>0
$$

which is satisfied if condition $(H)$ holds. The lemma is proved.

From now on, assume that condition $(H)$ holds. Then, for every $b \geq 0,(\Sigma)_{b}$ has a unique equilibrium point $e(b)$ in $K_{+}$. All the assumptions of Theorem 3.1 of [15. are satisfied. Therefore, $(\Sigma)_{b}$ is globally (with respect to initial states in $K$ ) asymptotically stable with respect to $e(b)$.

The next lemma studies the application $e:[0, c] \rightarrow K_{+}$that associates to $b \in$ $[0, c], e(b)$. Set $e_{n}:[0, c] \rightarrow \mathbb{R}_{+}$for the application that associates to $b$ the $n$-th coordinate of $e(b)$. Note that $\bar{x}_{n}$ is a fixed point of $e_{n}$.

Lemma 3. Assume that condition $(H)$ holds. Then

(1): $e_{n}$ is a strictly decreasing function and $\bar{x}_{n}$ is its unique fixed point;

(2): if $b_{1}<b_{2}$, then $e\left(b_{2}\right) \ll e\left(b_{1}\right)$;

(3): if $b<\bar{x}_{n}$, then $b<\bar{x}_{n}<e_{n}(b)$ and $\bar{x} \ll e(b)$; similarly if $\bar{x}_{n}<b$, then $e_{n}(b)<\bar{x}_{n}<b$ and $e(b) \ll \bar{x}$.

Proof of Lemma 3: Define $H(w, b):=h_{b}(w)$ for $(y, b) \in \mathbb{R}_{+}^{2}$. The differentiation of $H\left(e_{n}(b), b\right)=0$ yields

$$
\frac{d e_{n}}{d b}=-\frac{\partial_{b} H}{\partial_{y} H}=\frac{M^{\prime}\left(\varphi_{1}\left(z\left(e_{n}(b)\right)\right) \psi(b)\right) \varphi_{1}\left(z\left(e_{n}(b)\right)\right) \psi^{\prime}(b)}{h_{b}^{\prime}\left(e_{n}(b)\right)}<0,
$$

since $\psi$ is strictly decreasing. In addition, recall that $e_{1}(b)=z\left(e_{n}(b)\right)$ and $e_{k}(b)=$ $\varphi_{k}^{-1}\left(f_{k+1}\left(e_{k+1}(b)\right)\right)$ for $2 \leq k \leq n-1$. Then (2) holds since $z$ and the $\varphi_{k}^{-1} \circ f_{k+1}$ 's are strictly increasing functions.

Part (3) is immediate by taking into account the facts that the function $e_{n}$ is strictly decreasing, $\bar{x}_{n}$ is its unique fixed point and (2).

Lemma 4. Consider $\left(I^{l}\right)$ and $\left(S^{l}\right)$ the sequences of points of $K$ defined inductively as follows

$$
\left\{\begin{array}{l}
I^{l+1}=e\left(S_{n}^{l}\right), \\
S^{l+1}=e\left(I_{n}^{l}\right),
\end{array}\right.
$$

with $I^{0}=0$ and $S^{0}$ so that $e(0) \ll S^{0}$ and, for every $x \in T_{c}, x \ll S^{0}$. Here $e(0)$ is the equilibrium point of $(\Sigma)_{0}$. Then, for every $l \geq 0$, we have $I^{l} \ll \bar{x} \ll S^{l}$ and

$$
\lim _{l \rightarrow \infty} I^{l}=\lim _{l \rightarrow \infty} S^{l}=\bar{x},
$$

where $\bar{x}$ is the equilibrium point of $(\Sigma)$. 
Proof of Lemma 4; we first prove that for every $l \geq 0, I^{l} \ll \bar{x} \ll S^{l},\left(I^{l}\right)$ is increasing and $\left(S^{l}\right)$ is decreasing with respect to the partial order $\ll$. The argument goes by induction. More precisely, we show by induction that, for every $l \geq 0$, the next proposition $\left(P_{l}\right)$ holds,

$$
\left(P_{l}\right) I^{l} \ll \bar{x} \ll S^{l}, \quad I^{l} \ll I^{l+1}, \quad S^{l+1} \ll S^{l} .
$$

Clearly $\left(P_{0}\right)$ holds. Assume now that $\left(P_{l}\right), l \geq 0$ is true. Then $\bar{x}_{n}<S_{n}^{l}$. By Lemma 3, (3) we have

$$
I^{l+1}=e\left(S_{n}^{l}\right) \ll e\left(\bar{x}_{n}\right)=\bar{x}
$$

Similarly, we have $\bar{x} \ll S^{l+1}$. Since $I^{l} \ll I^{l+1}$, then $I_{n}^{l}<I_{n}^{l+1}$ and finally

$$
S^{l+2}=e\left(I_{n}^{l+1}\right) \ll e\left(I_{n}^{l}\right)=S^{l+1} .
$$

Similarly, $I^{l+1} \ll I^{l+2}$.

Since the sequences $\left(I^{l}\right)$ and $\left(S^{l}\right)$ are monotone (component-wise) they converge to $I$ and $S$ with $I \leq \bar{x} \leq S$. By passing to the limit in (26), we have

$$
I=e\left(S_{n}\right), \quad S=e\left(I_{n}\right),
$$

which implies that $I_{n}=e_{n}\left(S_{n}\right)$ and $S_{n}=e_{n}\left(I_{n}\right)$, i.e. $I_{n}$ and $S_{n}$ are fixed points of $e_{n} \circ e_{n}$. The derivative of that function is $\left.\frac{d e_{n}}{d b}\left(e_{n}\right)\right) \frac{d e_{n}}{d b} \geq 0$. If it is smaller than one then $\bar{x}_{n}$ is the unique fixed point of $e_{n} \circ e_{n}$ on $\mathbb{R}_{+}$and (27) is proved.

Finally to get $\left.\frac{d e_{n}}{d b}\left(e_{n}\right)\right) \frac{d e_{n}}{d b} \leq 1$, it is enough to have $\left|\frac{d e_{n}}{d b}\right| \leq 1$. Taking into account $(25)$, it is enough that, for every $b \in[0, c)$

$$
M^{\prime}\left(\varphi_{1}\left(z\left(e_{n}(b)\right)\right) \psi(b)\right) \varphi_{1}\left(z\left(e_{n}(b)\right)\right)\left|\psi^{\prime}(b)\right| \leq h_{b}^{\prime}\left(e_{n}(b)\right)
$$

which in turn follows from the next inequality

$$
M^{\prime}\left(\varphi_{1}\left(z\left(e_{n}(b)\right)\right) \psi(b)\right)\left(\varphi_{1}\left(z\left(e_{n}(b)\right)\right)+\frac{d \varphi(z(y))}{d y}\left(e_{n}(b)\right)\right) \leq 1 .
$$

Because an upper bound of $M^{\prime}$ is given in equation (12), $\varphi_{1}$ is bounded above by $a_{1}$, and $\frac{d \varphi(z(y))}{d y}$ by $c$, then $(\underline{30)})$ is implied by condition $(H)$. Since the latter already holds true, the proof of the lemma is finished.

Lemma 5. Let $x \in K$. Then $0 \ll \underline{\lim } \gamma_{x}$

Proof of Lemma 5: Without loss of generality, we take $0 \ll x$ (cf. Proposition 1, $(A))$ and argue by contradiction. Then there exists $1 \leq i \leq n$ so that $P_{i}$ holds:

$$
\left(P_{i}\right): \quad \forall \varepsilon>0, \forall t>0, \exists t^{\prime}>t, \quad x_{i}\left(t^{\prime}\right)<\varepsilon,
$$

where $x_{j}:=\left(\gamma_{x}\right)_{j}, 1 \leq j \leq n$.

Consider the largest interval $I$ containing $t^{\prime}$ so that $x_{i}(s) \leq \varepsilon$ for $s \in I$. By taking $\varepsilon$ small enough, the interval $I$ is of the type $\left[t_{0}, \cdots\right.$ with $t_{0}>0$. By continuity, $x_{i}\left(t_{0}\right)=\varepsilon$ and $\dot{x}_{i}\left(t_{0}\right) \leq 0$. Clearly, $i$ cannot be equal to 1 , otherwise, from $\dot{x}_{i}\left(t_{0}\right) \leq 0$, we would deduce that $c \leq a_{1} \varepsilon$, which is impossible for $\varepsilon$ small enough.

If $\left(P_{2}\right)$ holds, then $x_{2}\left(t_{0}\right)=\varepsilon$ and $\dot{x}_{2}\left(t_{0}\right) \leq 0$ imply that $x_{1}\left(t_{0}\right) \leq C_{1} \varepsilon$, with $C_{1}$ only depending on the positive parameters $a_{1}, a_{2}, k_{2}, k_{1}$. This means that $\left(P_{1}\right)$ holds and we have a contradiction.

Replacing 2 by any index $i \geq 3$ in the previous sentence indicates that if $\left(P_{i}\right)$ holds, then the same is true for $\left(P_{i-1}\right)$ and by a trivial induction we again have $\left(P_{1}\right)$. Therefore, if any $\left(P_{i}\right)$ holds, we get a contradiction. The proof of Lemma 5 is complete. 
4.2.2. Final part of the proof of Theorem 1. We are now ready to establish Theorem 1. From what precedes, the conclusion is the consequence of the next statement: for every $x \in K$ and for every $l \geq 0$

$$
\left(Q_{l}\right) \quad I^{l} \leq \underline{\lim } \gamma_{x} \leq \varlimsup \overline{\lim } \gamma_{x} \leq S^{l}
$$

Fix $x \in K$. Proposition $\left(Q_{l}\right)$ is proved inductively. For $l=0$, this is a consequence of Proposition 1, $(C)$. Applying Lemma 5, there exists $\varepsilon>0$ and $t_{0}(\varepsilon)>0$ such that for every $t \geq t_{0}(\varepsilon)$,

$$
\varepsilon v \leq \gamma_{x}(t) \leq S^{0}-\varepsilon v
$$

Then, passing to the limit we have

$$
e\left(S_{n}^{0}-\varepsilon\right) \leq \underline{\lim } \gamma_{x} \leq \varlimsup \lim \gamma_{x} \leq e(\varepsilon) .
$$

Note that, in equation (33), $\varepsilon$ may be replaced by any $0<\eta \leq \varepsilon$. Since $e$ is globally Lipschitz over $\mathbb{R}_{+}$, equation (34) implies $\left(Q_{1}\right)$ but also the existence of $t_{1}(\varepsilon)>0$ such that for every $t \geq t_{1}(\varepsilon)$,

$$
I^{1}+C_{1} \varepsilon v \leq \gamma_{x}(t) \leq S^{1}-C_{1} \varepsilon v,
$$

for some $0<C_{1} \leq 1$ independent of $\varepsilon$. Notice that equation (35) is of the same type as equation (33) and then leads to equations similar to (34) and again (35). In that way, we obtain, for every $l \geq 2$,

$$
e\left(S_{n}^{l}-C_{l} \varepsilon\right) \leq \underline{\lim } \gamma_{x} \leq \varlimsup \lim \gamma_{x} \leq e\left(I_{n}^{l}+C_{l} \varepsilon\right),
$$

and the existence of $t_{l}(\varepsilon)$ such that, for every $t \geq t_{l}(\varepsilon)$,

$$
I^{l}+C_{l+1} \varepsilon v \leq \gamma_{x}(t) \leq S^{l}-C_{l+1} \varepsilon v,
$$

with $C_{l+1} \leq C_{l} \leq 1$ independent of $\varepsilon$. Letting $\varepsilon$ tend to zero in (하) , we get $\left(Q_{l}\right)$.

4.3. General case. In this section, we will go back to system (1)-(2)-(3). A massbalance model for such a network has a unique equilibrium, as was shown in Section 3. In order to show the uniqueness of the equilibrium, we had separated the metabolites into three families: the root, the intermediate, and the boundary metabolites, which resulted in (1)-(2)-(3); in order to prove the stability of this equilibrium, it is needed to split the metabolites differently (for simplicity of notation, we will denote the elements of $\mathcal{P}(1)$ as $\left.\left\{k_{1}, \cdots, k_{r}\right\}\right)$ :

- The first equation, (1), is unchanged

$$
\dot{x}_{1}=c-\sum_{i=1}^{r} \varphi_{1 k_{i}}\left(x_{1}, x^{\left[k_{i}\right]}\right)-\mu x_{1}
$$

- For the products of $x_{1}$ (that we now denote $x_{k_{i}}$ ), the general equation, (2), is rewritten as

$$
\dot{x}_{k_{i}}=\varphi_{1 k_{i}}\left(x_{1}, x^{\left[k_{i}\right]}\right)-\sum_{j \in \mathcal{P}\left(k_{i}\right)} \varphi_{k_{i} j}\left(x_{k_{i}}\right)-\mu x_{k_{i}}
$$

- When $x_{k}$ is a product of $x_{l} \neq x_{1}$, the general equation (2) becomes

$$
\dot{x}_{k}=\varphi_{l k}\left(x_{l}\right)-\sum_{j \in \mathcal{P}(k)} \varphi_{k j}\left(x_{k}\right)-\mu x_{k}
$$

We impose the boundedness of the partial derivatives of $\varphi_{i j}$ in the following assumption: 
Assumption 3. The growth rate satisfies

$$
\mu>0
$$

and there exist $d_{i j} \geq 0, \alpha_{b}^{\left[k_{j}\right]} \geq 0$ such that

$$
\begin{aligned}
0 & \leq \frac{\partial \varphi_{i j}}{\partial x_{i}} \leq d_{i j} \quad \text { for all } i, j \\
-\alpha_{b}^{\left[k_{j}\right]} & \leq \frac{\partial \varphi_{1 k_{j}}}{\partial x_{b}^{\left[k_{j}\right]}} \leq 0 \quad \text { for all } j \leq r, b \leq n_{k_{j}}
\end{aligned}
$$

where $x_{b}^{\left[k_{j}\right]}$ is the inhibitor of the reaction $X_{1} \rightarrow X_{k_{j}}$, which lies at the index $b$ in the vector $x^{\left[k_{j}\right]}$.

Notation. From the arborescence structure, we know that there exists a unique path from $X_{1}$ to any metabolite $X_{s}$. This path takes the form $X_{1} \rightarrow X_{k_{j}} \rightarrow$ $\cdots X_{k} \rightarrow X_{l} \rightarrow \cdots \rightarrow X_{w} \rightarrow X_{s}$; if $X_{s}$ is an arbitrary metabolite, we will store the indices of this path (without 1 and $s$ ) in $C_{s}$; alternatively, if $x_{s}$ corresponds to some $x_{b}^{\left[k_{j}\right]}$, we will also denote this path $C_{b}^{\left[k_{j}\right]}$. Similarly, we denote $g_{s}(k)$ or $g_{b}^{\left[k_{j}\right]}(k)$ the index of the metabolite that follows $X_{k}$ in the path that connects $X_{1}$ to $X_{s}$.

This allows for the following theorem

Theorem 2. If Assumptions 1, 2, and 3 are satisfied and

$$
\sum_{j=1}^{r} \sum_{b=1}^{n_{k_{j}}}\left[\left(\frac{d_{1, k_{j}}}{\mu}+1\right) \prod_{k \in C_{b}^{\left[k_{j}\right]}} \frac{d_{k g_{b}^{\left[k_{j}\right]}(k)}}{\mu+d_{k g_{b}^{\left[k_{j}\right]}(k)}}\right]<\frac{\mu}{\max _{k_{s}, c} \alpha_{c}^{\left[k_{s}\right]}}
$$

and $\varphi_{1 k_{i}}$ is bounded for all $i \in\{1, \cdots, r\} \quad\left(0 \leq \varphi_{1 k_{i}} \leq B_{k_{i}}\right)$, then the equilibrium of system (37)-(38)-(39) is globally attractive in the positive orthant.

The proof of the above theorem follows exactly the same lines as that of Theorem 1 .

5. Limit cycles in metabolic networks with inhibition. In the previous section, we have shown that a wide class of models of branched metabolic networks has a single equilibrium and we have then proven that, under a small gain condition, this equilibrium is globally asymptotically stable and attractive. Obviously Condition $(40)$ is a only a sufficient condition. By performing simulations on simple examples, it is in fact easy to verify that Condition (40) is quite conservative and certainly not necessary. The stability of the equilibrium may be retained even if condition (40) is violated. But, in contrast, the global stability of the single equilibrium is not a generic property. As it is well known from the literature, if the inhibition becomes too strong, the system may be unstable and exhibit limit cycles. In this section we shall illustrate these properties with an example.

In this section, we will show that the answer to the second question is also "no": when condition (40) is not satisfied, the stability can be lost, so that we see that the stability of the metabolic networks is not a simple consequence of the structure of the models. Indeed, in this section, we shall exhibit an example where the equilibrium becomes unstable with a limit cycle (Hopf bifurcation) when condition $(40)$ is not satisfied. We will concentrate on the simplest case (8) of $n$ metabolites without branching and with sequential feedback inhibition, where we have replaced the exponential inhibition $\frac{1}{1+\alpha x_{n}}$ with a Hill function in the form $\frac{1}{1+\left(x_{n} / \theta_{n}\right)^{p}}$. This example is close to the classical Goodwin oscillator $([6])$. 
We can directly apply Theorem 2 to this system:

$$
\left\{\begin{array}{ccccc}
\dot{x}_{1} & = & & -\varphi_{1}\left(x_{1}, x_{n}\right) & -\mu x_{1} \\
\dot{x}_{2} & = & \varphi_{1}\left(x_{1}, x_{n}\right) & -\varphi_{2}\left(x_{2}\right) & -\mu x_{2} \\
\dot{x}_{3} & = & \varphi_{2}\left(x_{2}\right) & -\varphi_{3}\left(x_{3}\right) & -\mu x_{3} \\
\vdots & \vdots & \vdots & \vdots & \vdots \\
\dot{x}_{n} & = & \varphi_{n-1}\left(x_{n-1}\right) & -\varphi_{n}\left(x_{n}\right) & -\mu x_{n}
\end{array}\right.
$$

and condition (40) becomes

$$
\frac{\alpha}{\mu}\left(\frac{d_{1}}{\mu}+1\right) \prod_{k=2}^{n-1} \frac{d_{k}}{\mu+d_{k}}<1
$$

In order to try and give an answer to our question, we will study the local stability of the equilibrium of (41) when we do not impose condition (40), and see if it is generic. The Jacobian linearization of (41) has the following form:

$\mathcal{A}=\left(\begin{array}{ccccc}-\frac{\partial \varphi_{1}\left(x_{1}, x_{n}\right)}{\partial x_{1}}-\mu & 0 & \cdots & 0 & -\frac{\partial \varphi_{1}\left(x_{1}, x_{n}\right)}{\partial x_{n}} \\ \frac{\partial \varphi_{1}\left(x_{1}, x_{n}\right)}{\partial x_{1}} & -\varphi_{2}^{\prime}\left(x_{2}\right)-\mu & \cdots & 0 & \frac{\partial \varphi_{1}\left(x_{1}, x_{n}\right)}{\partial x_{n}} \\ 0 & \varphi_{2}^{\prime}\left(x_{2}\right) & \cdots & 0 & 0 \\ \vdots & \vdots & \vdots & \vdots & \vdots \\ 0 & 0 & \cdots & -\varphi_{n-1}^{\prime}\left(x_{n-1}\right)-\mu & 0 \\ 0 & 0 & \cdots & \varphi_{n-1}^{\prime}\left(x_{n-1}\right) & -\varphi_{n}^{\prime}\left(x_{n}\right)-\mu\end{array}\right)$

so that we can compute $\operatorname{det}(s I-\mathcal{A})$ by working on the first line

$$
\begin{gathered}
\operatorname{det}(s I-\mathcal{A})=\left(s+\frac{\partial \varphi_{1}\left(x_{1}, x_{n}\right)}{\partial x_{1}}+\mu\right)\left[\prod_{i=2}^{n}\left(s+\varphi_{i}^{\prime}\left(x_{i}\right)+\mu\right)\right. \\
\left.+(-1)^{n}\left(-\frac{\partial \varphi_{1}\left(x_{1}, x_{n}\right)}{\partial x_{n}}\right) \prod_{i=2}^{n-1}\left(-\varphi_{i}^{\prime}\left(x_{i}\right)\right)\right] \\
+(-1)^{n+1} \frac{\partial \varphi_{1}\left(x_{1}, x_{n}\right)}{\partial x_{n}} \frac{\partial \varphi_{1}\left(x_{1}, x_{n}\right)}{\partial x_{1}} \prod_{i=2}^{n-1}\left(-\varphi_{i}^{\prime}\left(x_{i}\right)\right) \\
=\left(s+\frac{\partial \varphi_{1}\left(x_{1}, x_{n}\right)}{\partial x_{1}}+\mu\right) \prod_{i=2}^{n}\left(s+\varphi_{i}^{\prime}\left(x_{i}\right)+\mu\right) \\
-\left(s+\frac{\partial \varphi_{1}\left(x_{1}, x_{n}\right)}{\partial x_{1}}+\mu\right) \frac{\partial \varphi_{1}\left(x_{1}, x_{n}\right)}{\partial x_{n}} \prod_{i=2}^{n-1} \varphi_{i}^{\prime}\left(x_{i}\right) \\
+\frac{\partial \varphi_{1}\left(x_{1}, x_{n}\right)}{\partial x_{n}} \frac{\partial \varphi_{1}\left(x_{1}, x_{n}\right)}{\partial x_{1}} \prod_{i=2}^{n-1} \varphi_{i}^{\prime}\left(x_{i}\right)
\end{gathered}
$$

It is easily seen that the last term compensates the term containing $\frac{\partial \varphi_{1}\left(x_{1}, x_{n}\right)}{\partial x_{1}}$ in the second term so that

$$
\begin{aligned}
& \operatorname{det}(s I-\mathcal{A})=\left(s+\frac{\partial \varphi_{1}\left(x_{1}, x_{n}\right)}{\partial x_{1}}+\mu\right) \prod_{i=2}^{n}\left(s+\varphi_{i}^{\prime}\left(x_{i}\right)+\mu\right) \\
&-(s+\mu) \frac{\partial \varphi_{1}\left(x_{1}, x_{n}\right)}{\partial x_{n}} \prod_{i=2}^{n-1} \varphi_{i}^{\prime}\left(x_{i}\right)=\left(s+\frac{\partial \varphi_{1}\left(x_{1}, x_{n}\right)}{\partial x_{1}}+\mu\right) \prod_{i=2}^{n}\left(s+\varphi_{i}^{\prime}\left(x_{i}\right)+\mu\right) \\
&+(s+\mu)\left|\frac{\partial \varphi_{1}\left(x_{1}, x_{n}\right)}{\partial x_{n}}\right| \prod_{i=2}^{n-1} \varphi_{i}^{\prime}\left(x_{i}\right)
\end{aligned}
$$

In order to evaluate the local stability of the equilibrium $\bar{x}$, we evaluate this polynomial in $x=\bar{x}$. The application of the Routh-Hurwitz criterion for $n=3$ shows that $\mathcal{A}$ is stable if $\frac{\partial \varphi_{1}\left(x_{1}, x_{n}\right)}{\partial x_{1}}+\mu>0, \varphi_{i}^{\prime}\left(x_{i}\right)+\mu>0$, and $\frac{\partial \varphi_{1}\left(x_{1}, x_{n}\right)}{\partial x_{n}} \leq 0$ at the equilibrium, so that the system is locally exponentially stable independently of condition (40). We then move to dimension 4. The application of the Routh-Hurwitz criterion shows that, if everything else stays the same, at least one root of $\operatorname{det}(s I-\mathcal{A})$ goes into the right-half plane if $\left|\frac{\partial \varphi_{1}\left(x_{1}, x_{n}\right)}{\partial x_{n}}\right|$ becomes large. 
We have then built an example exhibiting such a property: when the parameter present in the inhibition factor $(p)$ is modified, only $\left|\frac{\partial \varphi_{1}\left(x_{1}, x_{n}\right)}{\partial x_{n}}\right|$ is modified in $\operatorname{det}(s I-\mathcal{A})$ at the equilibrium: we consider

$$
\left\{\begin{array}{ccccc}
\dot{x}_{1} & = & & -\frac{1}{1+\left(x_{4} / 19\right)^{p}} \frac{3.2 x_{1}}{1+x_{1}} & -0.01 x_{1} \\
\dot{x}_{2} & = & \frac{1}{1+\left(x_{4} / 19\right)^{p}} \frac{3.2 x_{1}}{1+x_{1}} & -\frac{1.4 x_{2}}{1+x_{2}} & -0.01 x_{2} \\
\dot{x}_{3} & \frac{1.4 x_{2}}{1+x_{2}} & -\frac{1.2 x_{3}}{1+x_{3}} & -0.01 x_{3} \\
\dot{x}_{4} & = & \frac{1.2 x_{3}}{1+x_{3}} & -\frac{x_{4}}{1+x_{4}} & -0.01 x_{4}
\end{array}\right.
$$

which has the structure of (41) with $n=4$, and where we take $\varphi_{1}\left(x_{1}, x_{4}\right)=$ $\frac{1}{1+\left(x_{4} / 19\right)^{p}} \frac{3.2 x_{1}}{1+x_{1}}$ so that we can easily see that condition (42)

$$
\frac{\alpha}{\mu}\left(\frac{d_{1}}{\mu}+1\right) \frac{d_{2}}{\mu+d_{2}} \frac{d_{3}}{\mu+d_{3}}<1
$$

is only satisfied when $p=0$ (so that the inhibiting factor is constant at $\frac{1}{2}$ ).

The different forms of the inhibiting factor $\frac{1}{1+\left(x_{4} / 19\right)^{p}}$ are illustrated on Figure 43 where we see that $p$ strongly influences the maximal slope of the inhibition function. System (43) has a single equilibrium in $x=(19,19,19,19)^{T}$, and, at the equilibrium,

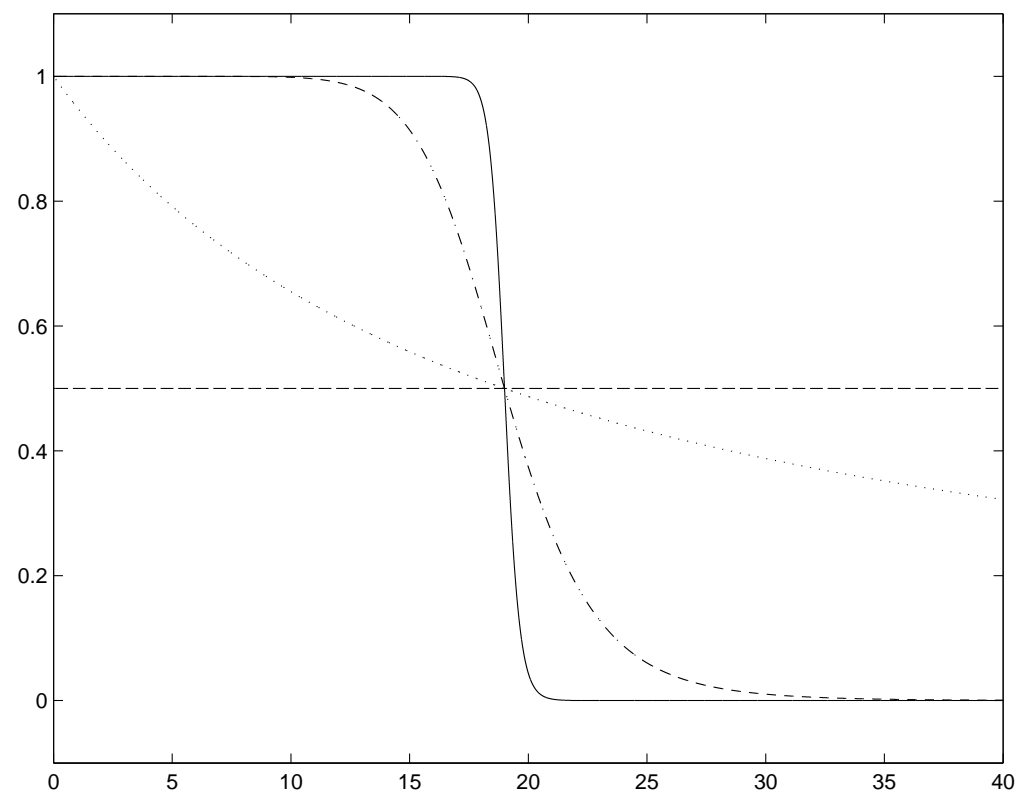

FiguRE 4. Form of the inhibiting factor $\frac{1}{1+\left(x_{4} / 19\right)^{p}}$ for $p=0$ (dashed-line), $p=1$ (dotted line), $\mathrm{p}=10$ (dash-dotted line) and $p=60$ (solid line)

we have

$\operatorname{det}(s I-\mathcal{A})=(s+0.0140)(s+0.0135)(s+0.0130)(s+0.0125)+4.210^{-7} p(s+0.01)$ which is stable for $p<56.4519$, and is not stable for $p$ larger than that value. This transition from a stable to an unstable equilibrium is illustrated on Figure 5 , where the time responses of the four states is illustrated for the value of $p=0$ 
(no inhibition), $p=10$ (weak inhibition), and $p=60$ (strong inhibition). In the latter case, oscillations appear. This corresponds to a limit cycle in the state-space. A Hopf bifurcation has taken place at $p=56.4519$. Despite this oscillation, the reaction rates for the three reactions $X_{2} \longrightarrow X_{3}, X_{3} \longrightarrow X_{4}$, and $X_{4} \longrightarrow \ldots$ are close to their maximum after the transient. The only limiting reaction is $X_{1} \longrightarrow X_{2}$ which, due to the inhibition is far from its maximum reaction rate.

The local stability of the equilibrium for $p<56.4519$ does not ensure global attractivity, but we have not noticed other behaviors than convergence towards the equilibrium when $p<56.4519$. This indicates that our result is very conservative: this is due to the fact that it comes from a small-gain analysis.
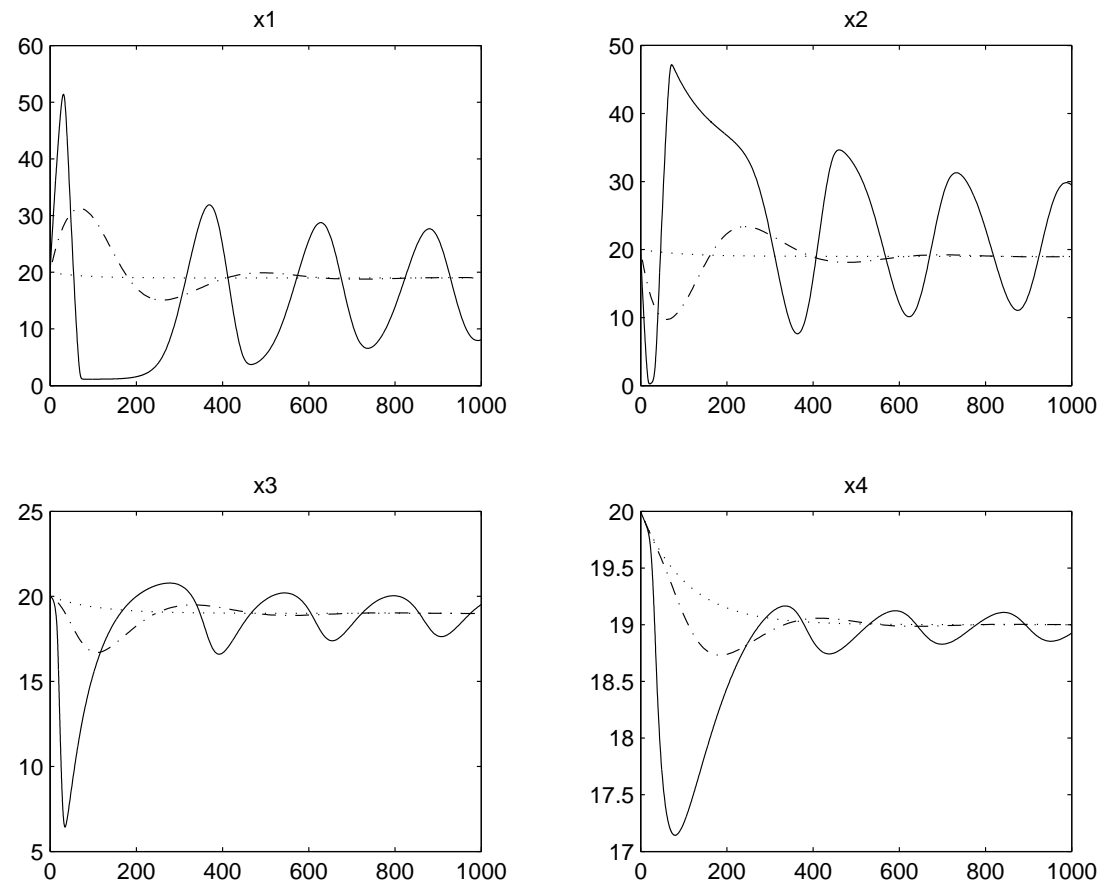

Figure 5. Evolution of the states of system (43) for $p=0$ (dotted line), $\mathrm{p}=10$ (dash-dotted line) and $p=60$ (solid line).

6. Conclusion. In this paper, we have shown that a large class of models of metabolic system only has a single equilibrium. We then have proven that, under a small gain condition, this equilibrium is globally attractive. Finally, with a simple example, we have illustrated the fact that the stability of the single equilibrium is not a generic property : the stability may be lost if the negative feedback inhibition is too strong.

\section{REFERENCES}

[1] R. Alves and M. Savageau, Systemic properties of ensembles of metabolic networks: application of graphical and statistical methods to simple unbranched pathways, Bioinformatics, 16 (6) (2000), 534- 547.

[2] D. Angeli and E.D. Sontag, Monotone control systems, IEEE Trans. Autom. Control, 48 (2003), 1684-1698. 
[3] C. Berding and G. Haubs, On the stability of equilibria in metabolic feedback systems, J. Math. Biol., 22 (3) (1985), 349-352.

[4] Y. Chitour, F. Grognard and G. Bastin G., Stability analysis of a metabolic model with sequential feedback inhibition, in Positive Systems, Proceedings of the First Multidisciplinary Symposium on Positive Systems (POSTA 2003), edited by Luca Benvenuti, Alberto De Santis and Lorenzo Farina, Lecture Notes on Control and Information Sciences vol. 294, SpringerVerlag, Heidelberg, 2003.

[5] W. A. Coppel, Stability and asymptotic behavior of differential equations, Heath Math. Mono., D. C. Heath Comp., 1965.

[6] Goodwin B., Temporal Organization in Cells: A Dynamical Theory of Cellular Control Processes, Academic Press, 1963.

[7] J.L. Gouzé, Positive and negative circuits in dynamical systems, Journal of Biological Systems, 6 (1998), 11-15.

[8] F. Grognard, Y. Chitour and G. Bastin, Equilibria and stability analysis of a branched metabolic network with feedback inhibition, Proceedings of the CAB9 conference, Nancy, March 2004, 171-176.

[9] S.P. Hastings, On the uniqueness and global asymptotic stability of periodic solutions for a third order system, Rocky Mountain J. Math., 7 (3) (1977), 513-538.

[10] A. Hunding A., Limit-cycles in enzyme-systems with nonlinear negative feedback, Biophys. Struct. Mech. 1 (1974) 47-54.

[11] Y. Li and J. Muldowney, Global Stability for the SEIR Model in Epidemiology, Math. Bio. 125 (1995), 155-164.

[12] J. Mallet-Paret and H.L. Smith, The Poincaré-Bendixson theorem for monotone cyclic feedback systems, J. Dynam. Differential Equations, 4 (1990), 367-421.

[13] A.I. Mees and P.E. Rapp, Periodic metabolic systems: oscillations in multiple-loop negative feedback biochemical control networks, J. Math. Biol., 5 (2) (1978), 99-114.

[14] E. Plahte, T. Mestl and S. Omholt, Feedback loops, stability and multistationarity in dynamical systems, Journal of Biological Systems, 3 (1995) 409-413.

[15] H.L. Smith, Monotone Dynamical Systems, An introduction to the theory of competitive and cooperative systems, Math. Surveys and Mono., Vol. 41, AMS, Providence, RI, 1995.

[16] E.H. Snoussi, Necessary conditions for multistationarity and stable periodicity, Journal of Biological Systems, 6 (1998), 3-9.

[17] G. Stephanopoulos, A. Aristidou and J. Nielsen, Metabolic Engineering: Principles and Methodologies, Academic Press, 1997.

[18] J.J. Tyson, On the existence of oscillatory solutions in negative feedback cellular control processes, J. Math. Biol., vol.1 (4) (1975), 311-315.

[19] H.E. Umbarger, Amino acid biosynthesis and regulation, Ann. Rev. Biochem., 47 (1978), 533-606.

Received July 2005; revised September 2005.

E-mail address: Yacine.Chitour@lss.supelec.fr

E-mail address: frederic.grognard@inria.fr

E-mail address: bastin@auto.ucl.ac.be 